\title{
Gravitational Waves from Coalescing Black Hole MACHO Binaries
}

\author{
Takashi Nakamura \\ Yukawa Institute for Theoretical Physics, Kyoto University, Kyoto 606 \\ Misao Sasaki and Takahiro Tanaka \\ Department of Earth and Space Science, Osaka University, Toyonaka 560 \\ and \\ Kip S. Thorne \\ Theoretical Astrophysics, California Institute of Technology, Pasadena, California 91125
}

\begin{abstract}
If MACHOs are black holes of mass $\sim 0.5 M_{\odot}$, they must have been formed in the early universe when the temperature was $\sim 1 \mathrm{GeV}$. We estimate that in this case in our galaxy's halo out to $\sim 50 \mathrm{kpc}$ there exist $\sim 5 \times 10^{8}$ black hole binaries whose coalescence times are comparable to the age of the universe, so that the coalescence rate will be $\sim 5 \times 10^{-2}$ events/year/galaxy. This suggests that we can expect a few events/year within $15 \mathrm{Mpc}$. The gravitational waves from such coalescing black hole MACHOs can be detected by the first generation of interferometers in the LIGO/VIRGO/TAMA/GEO network. Therefore, the existence of black hole MACHOs can be tested within the next five years by gravitational waves. ( submitted to Apj Letters April 11 1997)
\end{abstract}

Subject headings: gravitation — black holes — dark matter - — gravitational lensing - Galaxy: halo 


\section{Introduction}

The analysis of the first 2.1 years of photometry of 8.5 million stars in the Large Magellanic Cloud (LMC) by the MACHO collaboration (Alcock et al. 1996a suggests that $0.62_{-0.2}^{+0.3}$ of the halo consists of MACHOs of mass $0.5_{-0.2}^{+0.3} M_{\odot}$ in the standard spherical flat rotation halo model. The preliminary analysis of four years of data suggests the existence of at least four additional microlensing events with $t_{\text {dur }} \sim 9$ 90days in the direction of the LMC (Pratt 1997). The estimated masses of these MACHOs are just the mass of red dwarfs. However, the contribution of the halo red dwarfs to MACHO events should be small since the observed density of halo red dwarfs is too low (Bahcall et al. 1994, Graff \& Freese 1996a, Graff \& Freese 1996b). As for white dwarf MACHOs, the mass fraction of white dwarfs in the halo should be less than $10 \%$ since, assuming the Salpeter initial mass function (IMF), the bright progenitors of more white dwarfs than this would be in conflict with the number counts of distant galaxies (Charlot \& Silk 1995). If the IMF has a sharp peak around $2 M_{\odot}$, then the fraction could be $50 \%$ or so (Adams \& Laughlin 1996), sufficient to explain the MACHO observations. The existence of such a population of halo white dwarfs may or may not be consistent with the observed luminosity function Gould 1997, Lidman 1997, Freese 1997). In any case, future observations of high velocity white dwarfs in our solar neighborhood (Lidman 1997) will prove whether white dwarf MACHOs can exist or not.

If the number of high velocity white dwarfs turns out to be large enough to explain the MACHOs, then stellar formation theory should explain why the IMF is sharply peaked at $\sim 2 M_{\odot}$. If it is not, there arises a real possibility that MACHOs are absolutely new objects such as black holes of mass $\sim 0.5 M_{\odot}$ which could only be formed in the early universe, or boson stars with the mass of the boson $\sim 10^{-10} \mathrm{eV}$. Of course it is still possible that an overdense clump of MACHOs exists toward the LMC (Nakamura, Kanya \& Nishi 1996), MACHOs are brown dwarfs in the rotating halo (Spiro 1997) or MACHOs are stars in the thick disk (Turner 1997).

In this Letter we consider the case of black hole MACHOs (BHMACHOs). In this case, there must be a huge number (at least $\sim 4 \times 10^{11}$ ) of black holes in the halo and it is natural to expect that some of them are binaries. In $\S 2$ we estimate the fraction $f(a, e)$ dade of all BHMACHOs that are in binaries with semimajor axis $a$ in range $d a$ and eccentricity $e$ in $d e$. We then use this distribution to estimate two observable event rates: First (end of $\S 2$ ) the rate of microlensing events we should expect toward the LMC due to binaries with separation $\gtrsim 2 \times 10^{14} \mathrm{~cm}$; our result is in accord with the observation of one such event thus far (Bennett et al. 1996). Second ( $(3)$, the rate of coalescence of BHMACHO binaries out to $15 \mathrm{Mpc}$ distance. The gravitational waves from such coalescences should be detectable by the first interfereomters in the LIGO/VIRGO/TAMA/GEO network (Barish 1996, Brillet 1996, Tsubono 1996, Hough 1996), and our estimated event rate is a few events per year. In $\S 4$ we discuss some implications of our estimates.

\section{Formation of Solar Mass Black Hole MA- CHO Binaries}

Since it is impossible to make a black hole of mass $\sim 0.5 M_{\odot}$ as a product of stellar evolution, we must consider the formation of solar mass black holes in the very early universe (Yokoyama 1995, Jedamzik 1997). Our viewpoint here, however, is not to study detailed formation mechanisms, but to estimate the binary distribution that results.

The density parameter of BHMACHOs, $\Omega_{B H M}$, must be comparable to $\Omega_{b}$ (or $\Omega_{C D M}$ ) to explain the number of observed MACHO events. For simplicity, we assume that BHMACHOs dominate the matter energy density, i.e., $\Omega=\Omega_{B H M}$, though it is possible to consider other dark matter components in addition to BHMACHOs. To determine the mean separation of the BHMACHOs, it is convenient to consider it at the time of matter-radiation equality, $t=t_{e q}$. At this time, the energy densities of radiation and $\mathrm{BH}-$ MACHOs are approximately equal and are given by $\rho_{\text {eq }}=1.4 \times 10^{-15}\left(\Omega h^{2}\right)^{4} \mathrm{~g} / \mathrm{cm}^{3}$, where $h$ is the Hubble parameter in units of $100 \mathrm{~km} / \mathrm{s} / \mathrm{Mpc}$. Correspondingly, the mean separation of BHMACHOs with mass $M_{B H}$ at this time is given by

$$
\begin{aligned}
\bar{x} & =\left(M_{B H} / \rho_{e q}\right)^{\frac{1}{3}} \\
& =1.1 \times 10^{16}\left(M_{B H} / M_{\odot}\right)^{\frac{1}{3}}\left(\Omega h^{2}\right)^{-\frac{4}{3}} \mathrm{~cm} .
\end{aligned}
$$

We set the scale factor $R$ to unity at $t=t_{e q}$, so $\bar{x}$ can also be regarded as the comoving mean separation. Note that the Hubble horizon scale at $t=t_{e q}$ is $L_{e q} \sim$ $\sqrt{3 c^{2} / 8 \pi G \rho_{e q}}=1.1 \times 10^{21}\left(\Omega h^{2}\right)^{-2} \mathrm{~cm}$. 
During the radiation dominated era, the total energy inside the horizon increases as $R^{2}$. Since the Jeans mass in this era is essentially the same as the horizon mass, black holes are formed only at the time when the horizon scale is equal to the Schwarzschild radius of a BHMACHO. Thus the scale factor at the formation epoch becomes

$$
R_{f}=\sqrt{\frac{G M_{B H}}{c^{2} L_{e q}}}=1.2 \times 10^{-8}\left(\frac{M_{B H}}{M_{\odot}}\right)^{\frac{1}{2}}\left(\Omega h^{2}\right) .
$$

The age of the universe and the temperature $T_{f}$ at $R=R_{f}$ are $\sim 10^{-5}$ sec and $\sim 1 \mathrm{Gev}$, respectively.

As a foundation for computing the distribution function $f(a, e)$ for BHMACHO binaries, we assume that the BHMACHOs are created with a distribution of comoving separations $x$ that is uniform over the range from an initial physical separation equal to the black hole size (which turns out to be so small that for the computations that follow we can approximate it as zero) to a maximum separation $x=\bar{x}$. We also assume that the BHMACHOs' motions, if any, relative to the primordial gas have been redshifted to neglible speeds by the time their mutual gravitational attractions become important.

Consider a pair of black holes with mass $M_{B H}$ and a comoving separation $x<\bar{x}$. These holes' masses produce a mean energy density over a sphere the size of their separation given by $\rho_{B H} \equiv \rho_{e q}(\bar{x} / x R)^{3}$. This becomes larger than the radiation energy density $\rho_{r}=$ $\rho_{e q} / R^{4}$ for

$$
R>R_{m} \equiv(x / \bar{x})^{3} .
$$

This means that the binary decouples from the cosmic expansion and becomes a bound system when $R=R_{m}$. Note that the background universe is still radiation dominated at this stage.

If the motion of the two black holes is not disturbed, then the binary system cannot obtain any angular momentum so they coalesce to a single black hole on the free fall time scale. However, the tidal force from neighboring black holes gives the binary enough angular momentum to keep the holes from colliding with each other unless $x$ is exceptionally small.

We refer to the semimajor axis and the semiminor axis of the binary as $a$ and $b$, respectively, and we estimate $a$ as

$$
a=x R_{m}=x^{4} / \bar{x}^{3},
$$

We denote by $y$ the comoving separation of the nearest neighboring black hole from the center of mass of the binary. Then $b$ can be estimated as (tidal force $) \times(\text { free fall time })^{2}$

$$
b=\frac{G M_{B H} x R_{m}}{\left(y R_{m}\right)^{3}} \frac{\left(x R_{m}\right)^{3}}{G M_{B H}}=\left(\frac{x}{y}\right)^{3} a .
$$

Hence, the binary's eccentricity $e$ is given by

$$
e=\sqrt{1-(x / y)^{6}}
$$

Since (by assumption) $x$ and $y$ have uniform probability distributions in the range $x<y<\bar{x}$, the probability distribution of $a$ and $e$ is

$$
\begin{aligned}
f(a, e) d a d e & =18 x^{2} y^{2} \bar{x}^{-6} d x d y \\
& =(3 / 2) a^{\frac{1}{2}} \bar{x}^{-\frac{3}{2}} e\left(1-e^{2}\right)^{-\frac{3}{2}} d a d e
\end{aligned}
$$

From the condition that $y<\bar{x}$, the maximum value of the eccentricity for a fixed $a$ is given by $e_{\max }=$ $\sqrt{1-(a / \bar{x})^{\frac{3}{2}}}$. Integrating $f(a, e)$ with respect to $e$, we obtain the following distribution of the semimajor axis

$$
f_{a}(a) d a=\frac{3}{2}\left[\left(\frac{a}{\bar{x}}\right)^{\frac{3}{4}}-\left(\frac{a}{\bar{x}}\right)^{\frac{3}{2}}\right] \frac{d a}{a}
$$

From Eq. (8), it is found that the fraction of BHMACHOs that are in binaries with $a \sim 2 \times 10^{14} \mathrm{~cm}$ is $\sim 8 \%$ and $\sim 0.9 \%$ for $\Omega h^{2}=1$ and 0.1 , respectively. This estimated fraction of $\sim 10$ AU size BHMACHO binaries is slightly smaller than the observed rate of binary MACHO events (one binary event in 8 observed MACHOs), but the agreement is good given the statistics of small numbers.

\section{Gravitational Waves from Coalescing BH- MACHO Binaries}

We consider here short period BHMACHO binaries. Their coalescence times due to the emission of gravitational waves are approximately given by (Peters and Mathews 1963, Peters 1964)

$$
t=t_{0}\left(\frac{a}{a_{0}}\right)^{4}\left(1-e^{2}\right)^{\frac{7}{2}}
$$

where $t_{0}=10^{10}$ year and

$$
a_{0}=2 \times 10^{11}\left(\frac{M_{B H}}{M_{\odot}}\right)^{\frac{3}{4}} \mathrm{~cm}
$$

is the semimajor axis of a binary with circular orbit which coalesces in $t_{0}$. Integrating Eq. (7) for a fixed 
$t$ with the aid of Eq. (9), we obtain the probability distribution for the coalescence time $f_{t}(t)$ as

$$
f_{t}(t) d t=\frac{3}{29}\left[\left(\frac{t}{t_{\max }}\right)^{\frac{3}{37}}-\left(\frac{t}{t_{\max }}\right)^{\frac{3}{8}}\right] \frac{d t}{t},
$$

where $t_{\max }=t_{0}\left(\bar{x} / a_{0}\right)^{4}$. If the halo of our galaxy consists of BHMACHOs of mass $\sim 0.5 M_{\odot}, \sim 10^{12}$ BHMACHOs exist out to the LMC. The number of coalescing binary BHMACHOs with $t \sim t_{0}$ then becomes $\sim 5 \times 10^{8}$ for $\Omega h^{2}=0.1$ so that the event rate of coalescing binaries becomes $\sim 5 \times$ $10^{-2}$ events/year/galaxy. If, however, the BHMACHOs extend up to half way to M31, the number of coalescing binary BHMACHOs with $t \sim t_{0}$ can be $\sim 3 \times 10^{9}$ and the event rate becomes $\sim$ 0.3 events/year/galaxy. Both of these estimates are much larger than the best estimate of the event rate of coalescing neutron stars based on the statistics of binary pulsar searches in our galaxy, $\sim 1 \times$ $10^{-5}$ events/year/galaxy (Phinney 1991, Narayan, Piran \& Shemi 1991, Van den Heuvel \& Lorimer 1996).

The detectability of these waves by interferometers is most easily discussed in terms of the waves' "characteristic amplitude" $h_{c}$ (Eq. (46b) of Thorne 1987, with a well-known factor 2 correction):

$h_{c}=4 \times 10^{-21}\left(\frac{M_{\text {chirp }}}{M_{\odot}}\right)^{5 / 6}\left(\frac{\nu}{100 H z}\right)^{-1 / 6}\left(\frac{r}{20 M p c}\right)^{-1}$.

Here $M_{\text {chirp }}=\left(M_{1} M_{2}\right)^{3 / 5} /\left(M_{1}+M_{2}\right)^{1 / 5}$ is the "chirp mass" of the binary whose components have individual masses $M_{1}$ and $M_{2}$. This $h_{c}$ is to be compared with an interferometer's "sensitivity to bursts" $h_{S B}=11\left[f S_{h}(f)\right]^{1 / 2}$, where $S_{h}(f)$ is the spectral density of the interferometer's strain noise (cf. Eq. (111) of Thorne 1987, where $h_{S B}$ is denoted $\left.h_{3 / y r}\right)$. This $h_{S B}(f) \equiv h_{3 / y r}(f)$ is plotted in various publications, e.g. Abramovici et. al. (1989) and Thorne (1995). It has a minimum (optimal sensitivity) at a frequency $f \simeq 100 \mathrm{~Hz}$. For the first LIGO and VIRGO interferometers, which are expected to be operational in 2001 , that minimum is $h_{S B \min } \simeq 3 \times 10^{-21}$. The GEO600 and TAMA interferometers, with their somewhat shorter armlengths, will have $h_{S B m i n}$ a little worse than this in 2001. LIGO/VIRGO should be able to detect coalescing binaries, with high confidence, out to the distance for which $h_{c}=h_{S B \min }$ at the optimal frequency $f \simeq 100 \mathrm{~Hz}$. Inserting $M_{1}=M_{2} \simeq 0.5 M_{\odot}$ for BHMACHOs into the above equations, we see that the first LIGO/VIRGO interferometers in 2001 should be able to see BHMACHO coalescences out to about $15 \mathrm{Mpc}$ distance, i.e., out to the VIRGO cluster, where our estimates $(\sim 1 / 100$ years in each galaxy like our own) suggest an event rate of several per year.

LIGO R\&D for the first interferometers is now nearing completion and is beginning to be redirected toward interferometer enhancements, for which the sensitivity goal is a factor 10 improvement, to $h_{S B \min } \simeq$ $3 \times 10^{-22}$ (Barish et. al. 1996). In the mid 2000s, with these enhancements in place, LIGO should be able to see BHMACHO coalescences out to about $150 \mathrm{Mpc}$, which would give a few events per year even if the event rate is 1000 times smaller than our estimates, $\sim 10^{-5}$ events/year/galaxy.

\section{Discussion}

In this Letter we have estimated the distribution function of binary BHMACHOs in order of magnitude. It is possible to compute the distribution function more accurately by N-body numerical simulations. This is an important, challenging numerical problem.

Our estimated event rate for coalescing BHMA$\mathrm{CHO}$ binaries is comparable to or greater than the most optimistic upper limit for binary neutron star coalescences (Phinney 1991), which are one of the most important sources of gravitational waves. Coalescing neutron stars are also regarded as possible sources of the gamma-ray bursts (Meszaros 1995). If so, then the detection of gravitational waves should be accompanied by a gamma-ray burst. If we consider the fire ball model (Meszaros 1995) the time delay between the gravitational waves and the gamma-rays should be $\sim 1$ sec. By contrast, in the coalescence of binary BHMACHOs the emission of gamma-rays is not expected. This may enable us to distinguish coalescing binary BHMACHOs from coalescing binary neutron stars.

If gamma-rays are not emitted by coalescing binary neutron stars, we may still use their observed chirp masses $M_{\text {chirp }}$ to distinguish them from BHMACHO binaries. The chirp mass can be measured from the gravitational waves to a fraction of a per cent accuracy, which is much less than the expected spreads of BHMACHO masses and neutron star masses. The masses of neutron stars in binaires are expected to be $\sim 1.4 M_{\odot}$ corresponding to a chirp mass of $1.2 M_{\odot}$. 
This is supported observationally as well as theoretically since the mass of the iron core before the collapse is $\sim 1.4 M_{\odot}$.

Therefore if the chirp masses of BHMACHO binaries are much smaller than $1 M_{\odot}$ it is possible to identify them. However if the mass of a BHMACHO is $\sim 1 M_{\odot}$, coalescing BHMACHO binaries and coalescing neutron star binaries may be indistinguishable from the detected gravitational waves. In principle, however, black holes absorb some of the gravitational waves so that the evolution of the binary in the last few minutes (Cutler et al. 1993) is slightly different from that of a neutron star binary of the same mass. The difference arises in the 2.5th post-Newtonian order for the Kerr black hole case (Tagoshi 1997) and 4th order for the Schwarzschild black hole case (Poisson \& Sasaki 1995). This difference might be detectable, though it will present a challenging problem for gravitational wave data analysis.

It is known that the Silk damping of density perturbations on small scales causes distortions of the CMB spectrum by dumping acoustic energy into heat and thence into the CMB (Daly 1991, Barrow \& Coles 1991, Hu, Scott \& Silk 1994). Thus it is important to examine if the large primordial density perturbations that are needed for BHMACHO formation are compatible with the observed upper limit of CMB spectral distortions (Mather et al. 1994).

For a rms amplitude of density perturbations $\delta(l)$ on a comoving scale $l$ in the radiation dominated era, the fraction of the universe that turns into black holes is given by $f(l) \approx \delta(l) \exp \left(-1 / 18 \delta(l)^{2}\right)$ Carr 1975). In the present case, $f(l)$ is equal to $R_{f}$ given by Eq. (2) and $l$ is equal to the comoving scale $l_{B H}$ of the density perturbations that give rise to BHMACHOs. Recalling that we fixed the scale factor $R$ to unity at the time $t_{e q}$ of matter-radiation equality, the present comoving scale corresponding to $l_{B H}$ is given by $\left(1+z_{e q}\right) l_{B H}=\left(1+z_{e q}\right) R_{f}^{-1} G M_{B H} c^{-2}=$ $5 \times 10^{17}\left(M_{B H} / M_{\odot}\right)^{1 / 2} \mathrm{~cm}$, where $z_{e q}$ is the redshift at $t=t_{\text {eq }}$. Since $R_{f} \sim 10^{-8}$, we need $\delta\left(l_{B H}\right)^{2} \sim$ $4 \times 10^{-3}$.

On the other hand, $\mathrm{Hu}$, Scott and Silk (1994) showed that the observational limit constrains the rms amplitude of density perturbations at horizon entry to be $\delta(l)^{2} \lesssim 10^{-4}$ for $l>l_{D}$, where $l_{D}$ is the comoving scale of the Silk damping at the double Compton thermalization time, which at present is given by $\left(1+z_{e q}\right) l_{D}=3 \times 10^{20} \mathrm{~cm}$. Hence the scale of in- terest $l_{B H}$ is approximately 3 orders of magnitude smaller than the scale $l_{D}$ to which the observational constraint applies.

Correspondingly, if we assume the primordial density perturbation spectrum to have a power-law shape, $P(k) \propto k^{n}$, on scales greater than $l_{B H}$, the above result implies that $n \gtrsim 1.6$. Interestingly, this value is consistent with the one suggested by the observed CMB anisotropies (Bennett et al. 1994) and it is marginally consistent with the COBE normalization (Hu, Scott \& Silk 1994). It may be that this rather blue spectrum can be produced by a variant of the socalled hybrid model of inflation Garcia-Bellido, Linde \& Wands 1996).

This work was supported in part by a Japan-US cooperative program on gravitational waves from coalescing binary compact objects (JSPS grant EPAR/138 $\&$ NSF grant INT-9417348). The main part of the paper was completed while the Japanese authors (TN, MS and TT) were visiting Caltech. This work was also supported by a Grant-in-Aid for Basic Research of the Ministry of Education, Culture, and Sports No.08NP0801,09640351 and by NSF grant AST-9417371.

\section{REFERENCES}

Adams, F.C. \& Laughlin, G. 1996 ApJ, 468, 586

Alcock, C. et al. 1996, astro-ph/9606165

Abramovici, A. et. al., Science, 256, 325.

Bahcall, J. N., Flynn, C., Gould, A., \& Kirhakos, S. 1994, ApJ, 435, L51

Barish, B. , Talk at TAMA Workshop on Gravitational Wave Detection, (November 12-14 1996 Saitama, Japan)

Barish,B. \& Sanders, G. et. al., LIGO Advanced R and D Program Proposal, Caltech/MIT, unpublished.

Barrow, J. D. \&Coles, P. 1991, MNRAS, 248, 52

Bennett, C. L. et al. 1994, ApJ, 464, L1

Bennett, D. P. et al. 1996, astro-ph/9606012

Brillet, A. , Talk at TAMA Workshop on Gravitational Wave Detection, (November 12-14 1996 Saitama, Japan) 
Carr, B. J. 1975, ApJ, 201, 1

Charlot, S. \& Silk, J. 1995, ApJ, 445, 124

Cutler, C. et al. 1993, Phys. Rev. Lett., 70, 2984

Daly, R. A. 1991, ApJ, 371, 14

Freese, K. , Talk at 3rd Microlensing Workshop (March 6-8 1997 Notre Dame University)

Garcia-Bellido, J., Linde, A. D. \& Wands, D., astro$\mathrm{ph} / 9605094$

Gould, A. , Talk at 3rd Microlensing Workshop (March 6-8 1997 Notre Dame University)

Graff, D.S. \& Freese, K. 1996a, ApJ, 456, L49

Graff, D.S. \& Freese, K. 1996b, ApJ, 467, L65

Jedamzik, K. , Talk at 3rd Microlensing Workshop (March 6-8 1997 Notre Dame University)

Hough, J. , Talk at TAMA Workshop on Gravitational Wave Detection, (November 12-14 1996 Saitama, Japan)

Hu, W., Scott, D. \& Silk, J. 1994, ApJ, 430, L5

Lidman, C. , Talk at 3rd Microlensing Workshop (March 6-8 1997 Notre Dame University)

Mather, J. C., et al. 1994, ApJ, 420, 439

Mészáros, P. 1995, in The 17th Texas Symposium on Relativistic Astrophysics, and references therein.

Nakamura, T. , Kan-ya, Y. \& Nishi, R. 1996, ApJ, 473, L99

Narayan, R. Piran, T. \& Shemi, A. 1991, ApJ, 379, L17

Peters, P.C. \& Mathews, J. 1963, Phys. Rev., 131, 435

Peters, P.C. 1964, Phys. Rev., 136, B1224

Phinney, E. S. 1991,ApJ, 380, L17

Poisson, E. \& Sasaki, M. 1995, Phys. Rev. D, 51, 5753

Pratt, M., Talk at 3rd Microlensing Workshop (March 6-8 1997 Notre Dame University)

Spiro, M., Talk at 3rd Microlensing Workshop (March 6-8 1997 Notre Dame University)
Tagoshi, H. 1997, Private communication.

Thorne, K.S. in 300 Years of Gravitation, eds. S. W. Hawking and W. Israel, Cambridge U. Press, p. 330.

Thorne,K.S. in Proceedings of the Snowmass 95 Summer Study on Particle and Nuclear Astrophysics and Cosmology, eds. E.W. Kolb and R. Peccei, p. 398.

Tsubono, K. , Talk at TAMA Workshop on Gravitational Wave Detection, (November 12-14 1996 Saitama, Japan)

Turner, M., Talk at 3rd Microlensing Workshop (March 6-8 1997 Notre Dame University)

Van den Heuvel, E.P.J. and Lorimer, D.R., MNRAS, 283, L37

Yokoyama, J. astro-ph 9509027

This 2-column preprint was prepared with the AAS LATEX macros v4.0. 